\title{
Oxaliplatin-Based Platinum(IV) Prodrug Bearing Toll-like Receptor 7 Agonist for Enhanced Immunochemotherapy
}

Li Tang, ${ }^{\dagger, \neq}$ Demin Cai, ${ }^{\dagger}$ Mian Qin, ${ }^{\dagger}$ Shuo Lu, ${ }^{\dagger}$ Ming-Hao Hu, ${ }^{\dagger}$ Shuangchen Ruan, ${ }^{\ddagger}$ Guangyi Jin, ${ }^{*,+\S, " ~ a n d ~ Z h i g a n g ~ W a n g *,+, " ~}$

†School of Pharmaceutical Sciences, Health Science Center, ${ }^{\ddagger}$ Key Laboratory of Optoelectronic Devices and Systems of Ministry of Education and Guangdong Province, College of Optoelectronic Engineering, IInternational Cancer Center, Health Science Center, and "The National-Regional Engineering Lab for Synthetic Biology of Medicine, Health Science Center, Shenzhen University, Shenzhen 518060, P. R. China 


\section{Supplemental results}<smiles></smiles>

oxaliplatin

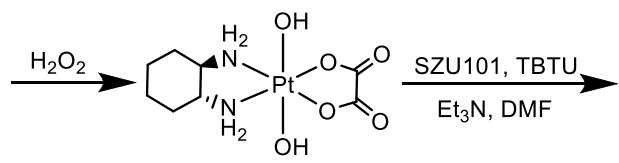

$\mathrm{Pt}(\mathrm{DACH})(\mathrm{OH})_{2} \mathrm{OX}$

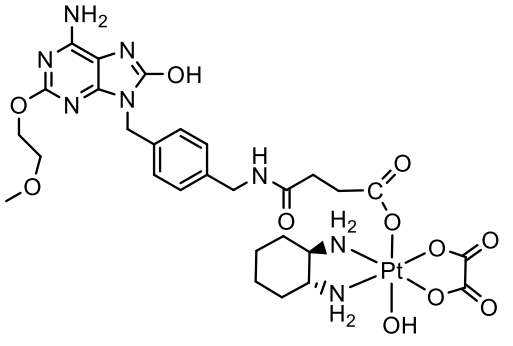

TPt

Scheme S1. Synthetic route of TPt.

A

A

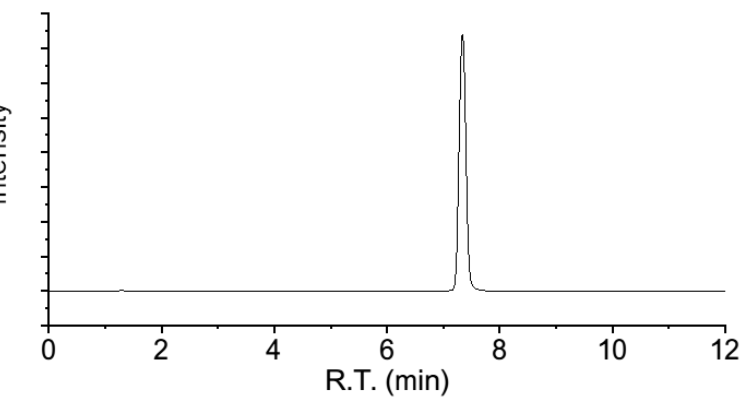

B

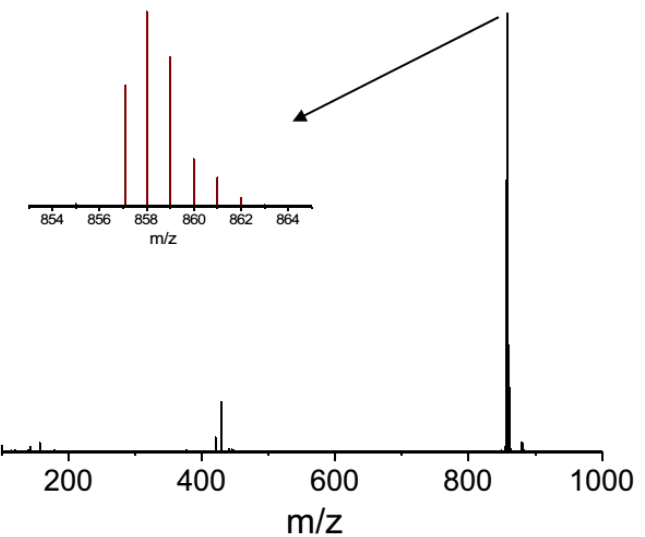

C

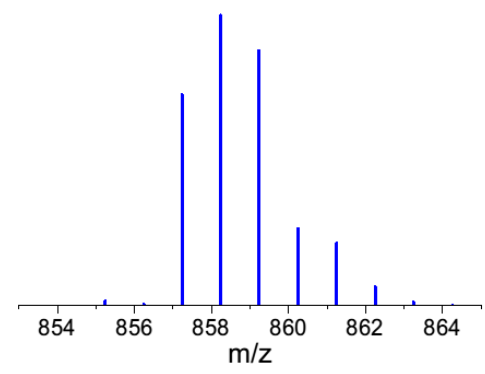

Figure S1. LC-MS analysis of TPt. (A). HPLC chromatograph (254 nm) of TPt ; (B). Mass spectra(ESI, positive mode) at the peak of panel $A$, which correspond to $T P t, m / z:[M+H]+$ calcd. for $\mathrm{C}_{28} \mathrm{H}_{39} \mathrm{~N}_{8} \mathrm{O}_{11} \mathrm{Pt} 858.25$, found 858.0.; (C). Stimulated mass spectra of [TPt+H] 


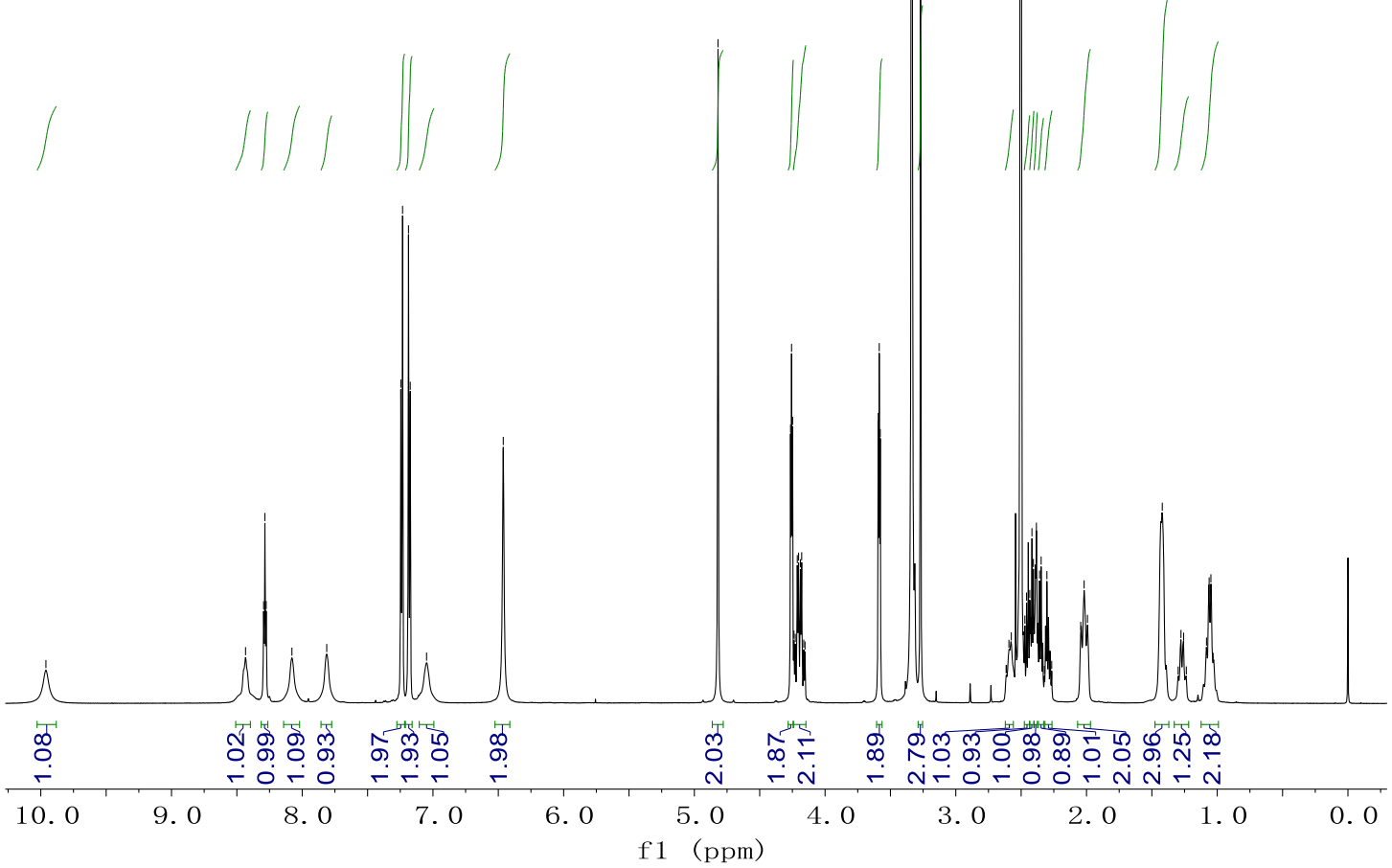

Figure S2. ${ }^{1} \mathrm{H}$ NMR spectrum of TPt in DMSO- $d_{6}$

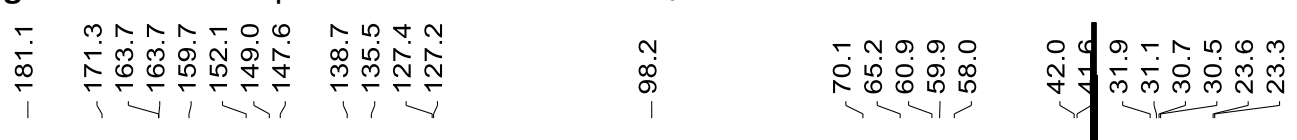

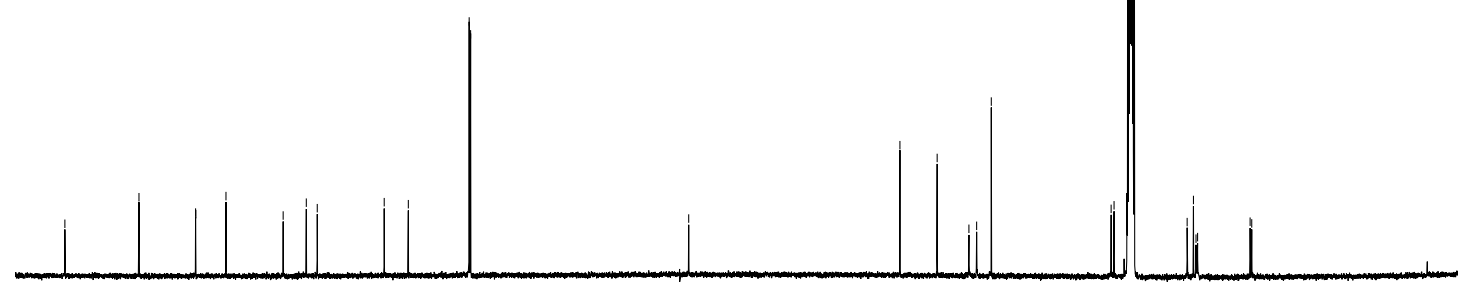

$\begin{array}{lllllllllllllllllll}180 & 170 & 160 & 150 & 140 & 130 & 120 & 110 & 100 & 90 & 80 & 70 & 60 & 50 & 40 & 30 & 20 & 10 & 0\end{array}$

Figure S3. ${ }^{13} \mathrm{C}$ NMR spectrum of TPt in DMSO- $d_{6}$ 


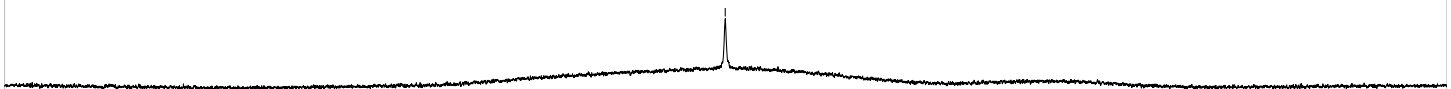

\begin{tabular}{|c|c|c|c|c|c|c|c|c|c|}
\hline 1950 & 1850 & 1750 & 1650 & 1550 & $\begin{array}{c}1450 \\
\mathrm{f} 1\end{array}$ & $\begin{array}{l}1350 \\
\mathrm{opm})\end{array}$ & 1250 & 1150 & 105 \\
\hline
\end{tabular}

Figure S4. ${ }^{195} \mathrm{Pt}$ NMR spectrum of TPt in DMSO-d $d_{6}$

213 \#16 RT: 0.22 AV: 1 NL: $4.60 E 7$

T: FTMS + p ESI Full ms [150.00-2000.00]

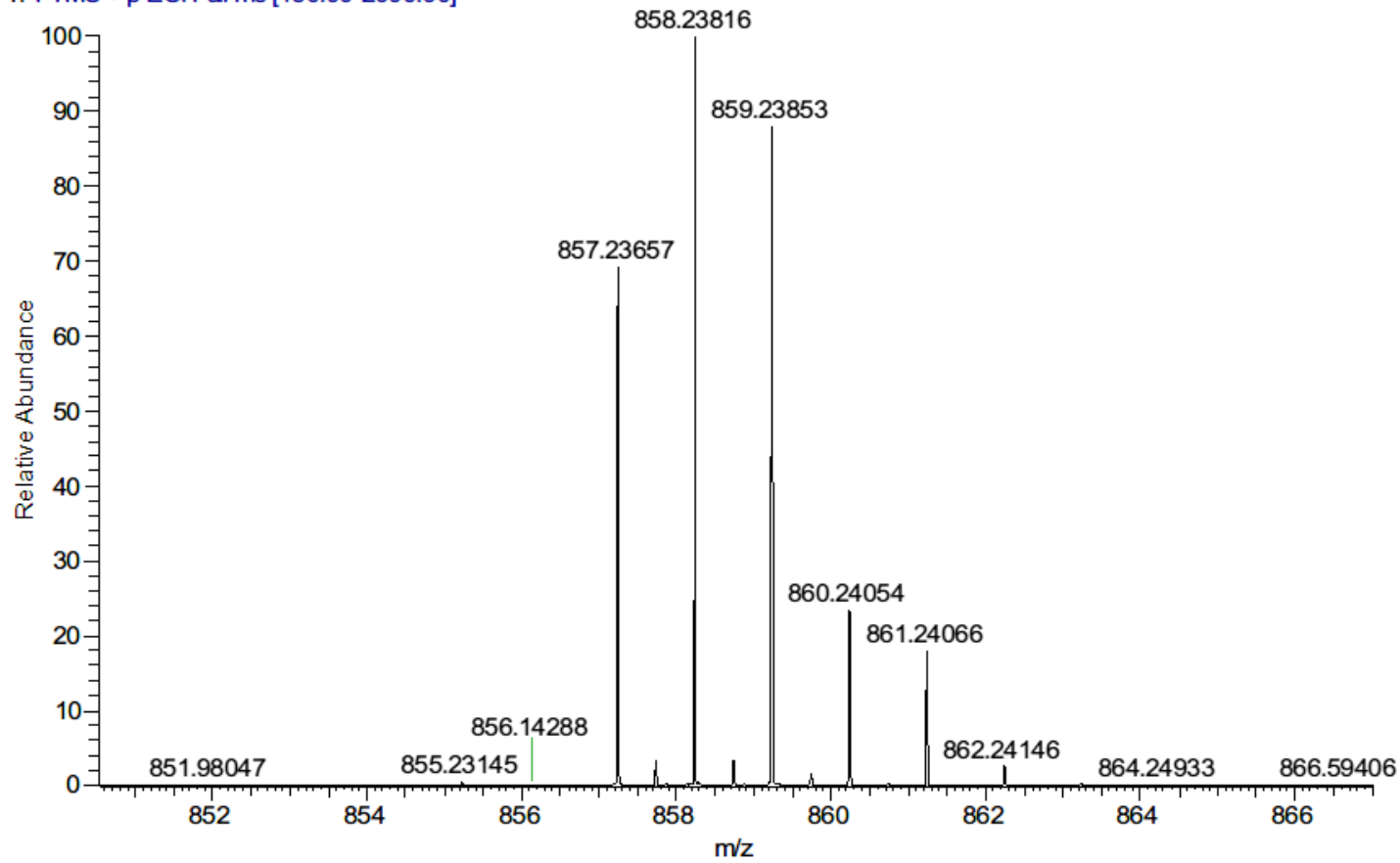

Figure S5. High resolution ESI-MS spectrum of TPt (positive mode). $m / z$ : $[\mathrm{M}+\mathrm{H}]^{+}$calcd. for $\mathrm{C}_{28} \mathrm{H}_{39} \mathrm{~N}_{8} \mathrm{O}_{11} \mathrm{Pt} 858.23805$, found 858.23816. 

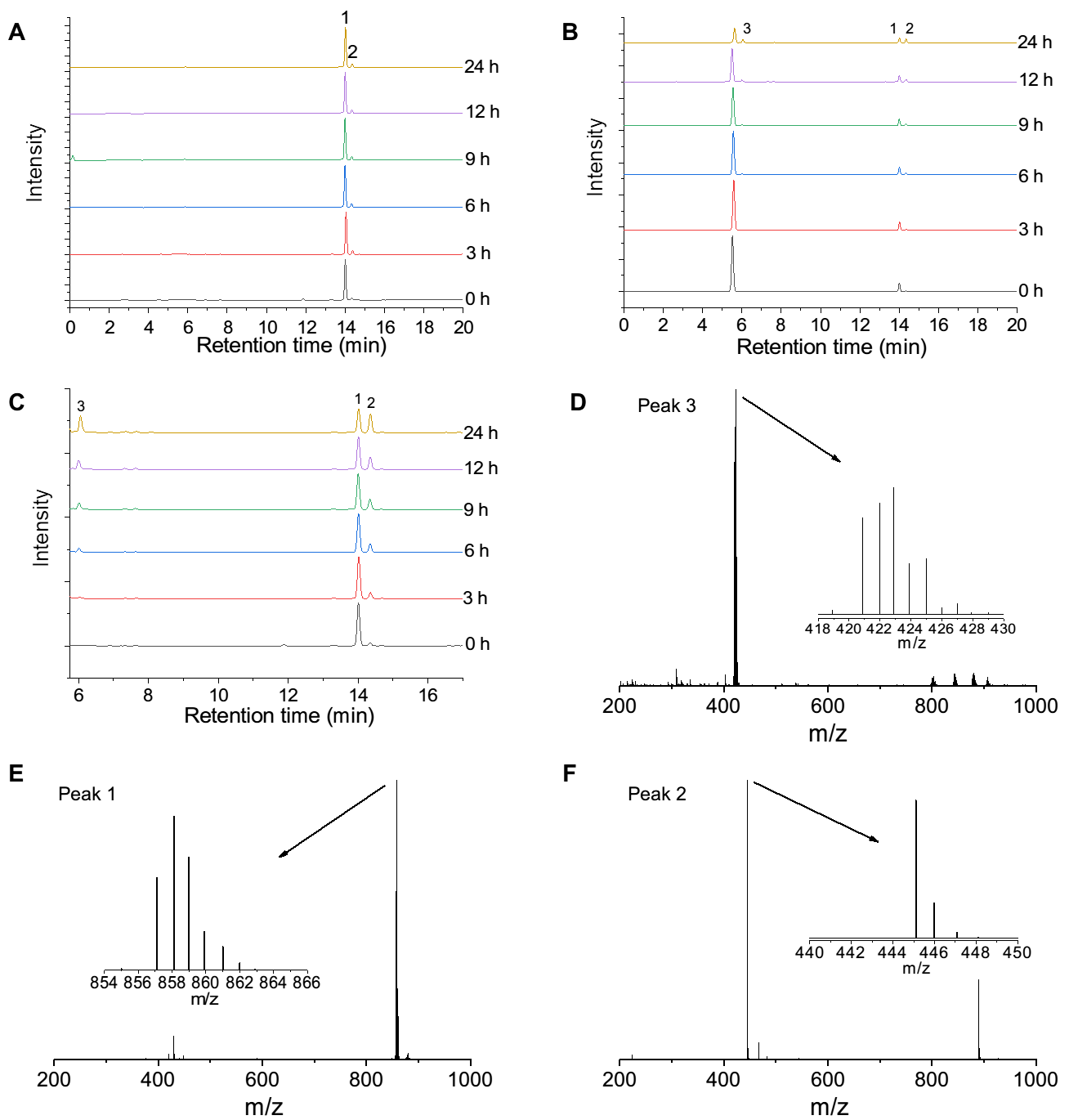

Figure S6. LC-MS analysis of the stability TPt in PBS buffer with or without sodium ascorbate.. (A). HPLC curve $(254 \mathrm{~nm})$ of TPt $(100 \mu \mathrm{M})$ in PBS at $37 \mathrm{C}$ different incubation time, ${ }^{1}$ peak of TPt, ${ }^{2}$ peak of SZU101; (B). HPLC curve $(254 \mathrm{~nm})$ of TPt $(100 \mu \mathrm{M})$ in PBS with the presence of $1 \mathrm{mM}$ sodium ascorbate at $37 \mathrm{C}$ after different incubation time. ${ }^{1}$ peak of TPt, ${ }^{2}$ peak of SZU101, ${ }^{3}$ peak of oxaliplatin (C). Enlaged figure of panel B from time $5.7 \mathrm{~min}$ to $17 \mathrm{~min}$ to get rid of ascorbate's peak. . ${ }^{1}$ peak of TPt, ${ }^{2}$ peak of SZU101, ${ }^{3}$ peak of oxaliplatin (D). MS spectra of peak 3 (Retention time at $5.99 \mathrm{~min}$ ), which correspond to [oxaliplatin+Na] ${ }^{+}$, the data was from sample TPt with sodium ascorbate after $24 \mathrm{~h}$ incubation. (E). MS spectra of peak 1 (Retention time $14.03 \mathrm{~min}$ ), which correspond to $[\mathrm{TPt}+\mathrm{H}]^{+}$, the data was from sample TPt with sodium ascorbate after $24 \mathrm{~h}$ incubation. (F). MS spectra of peak 3 (Retention time at $5.99 \mathrm{~min}$ ), which correspond to [SZU101+H] ${ }^{+}$, the data was from sample TPt with sodium ascorbate after $24 \mathrm{~h}$ incubation. 
A

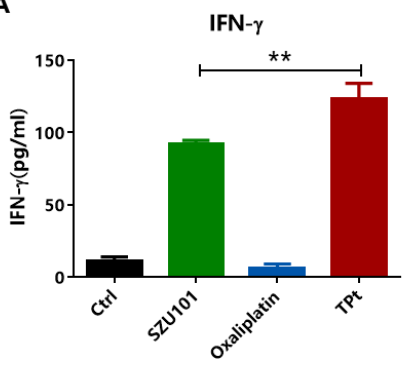

IL-12

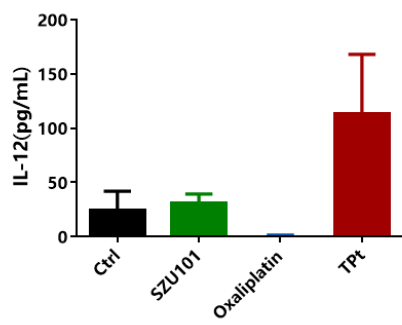

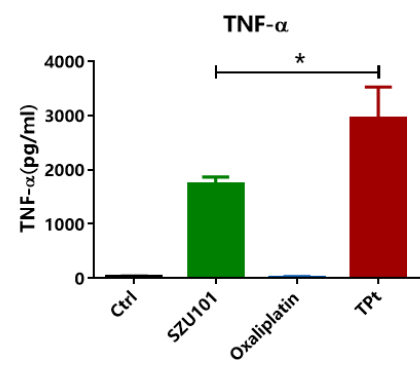

$E$

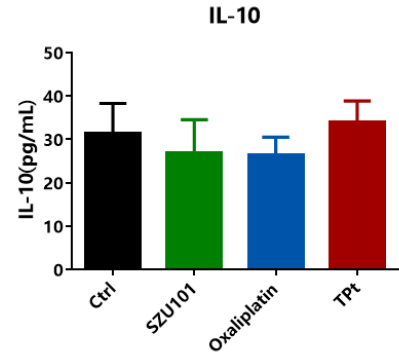

C

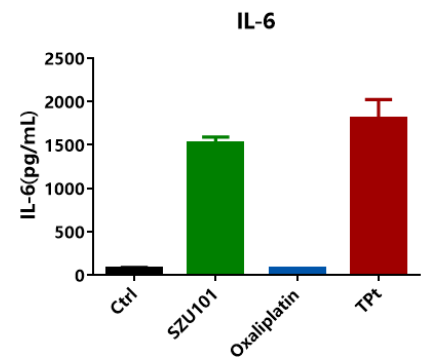

Figure S7. cytokine levels in the culture media of BMDCs treated with SZU101 $(5 \mu \mathrm{M})$, oxaliplatin $(5 \mu \mathrm{M})$, or TPt $(5 \mu \mathrm{M})$ for $18 \mathrm{~h}$. (A)IFN- $\gamma$; (B)TNF- $\alpha$; (C) IL-6; (D) IL-12; (E) IL-10. $n=3$. 